\title{
El antiplatonismo del Tractatus de Wittgenstein
}

\author{
Sílvio Pinto
}

\section{I}

\section{ntroducción}

Los intentos por eliminar los objetos en el análisis semántico del discur-

so matemático se han enfrentado usualmente al problema de cómo dar cuenta de la aparente objetividad de las matemáticas. Las soluciones antiplatonistas a esta cuestión varían considerablemente. Los intuicionistas, por ejemplo, han identificado la verdad matemática con la noción de prueba intuitiva. Afirman que una oración matemática es verdadera, si y sólo si puede encontrarse o reconocerse una prueba intuitiva de ella. Un problema con esta concepción podría ser que la verdad y la objetividad se conviertan en algo que depende de los juicios. Los convencionalistas, por su parte, han mantenido que la objetividad de las matemáticas puede explicarse en términos de la noción de regla sintáctica: ellos afirmarían que los enunciados lógicos y matemáticos son reglas de la sintaxis de nuestro lenguaje. Pero agregarían que estas reglas son estipulaciones (o convenciones). No obstante, tal concepción está en conflicto con fuertes intuiciones acerca de la objetividad y del carácter no arbitrario de las matemáticas y de la lógica.

En este trabajo me ocuparé del antiplatonismo de Wittgenstein. Mi propósito es investigar si acaso su primera filosofía de las matemáticas puede dar cuenta satisfactoriamente de la objetividad y el conocimiento matemáticos. Primero examinaré la solución de Wittgenstein al problema de la objetividad en las matemáticas. Puesto que considera que las matemáticas consisten en seguir reglas, su perspectiva debe explicar el carácter objetivo de las reglas matemáticas. En cuanto al conocimiento matemático se refiere, Wittgenstein debe reconciliar el aparente carácter normativo de las reglas matemáticas con

N. de la R.: Artículo escrito originalmente en inglés. Traducción de Efraín Lazos. 
su aplicación generalizada en la física, el lenguaje ordinario y otros dominios. Esto lo llevará a mantener que el conocimiento de las reglas matemáticas debe ser a priori. Además del valor exegético de clarificar la explicación tractariana de las matemáticas, este ejercicio arroja luz sobre la concepción de las matemáticas del Wittgenstein maduro.

\section{Reglas tractarianas}

La hostilidad de Wittgenstein frente al platonismo matemático se remonta al Tractatus. ${ }^{1}$ Ya en este primer libro encontramos la afirmación de que una explicación de la aplicación de las matemáticas no requiere postular objetos matemáticos. El argumento tractariano contra el platonismo se basa en consideraciones de uso. La siguiente es quizás la formulación más explícita.

No es, pues, nunca, en la vida, una proposición matemática lo que nosotros necesitamos, sino que utilizamos las proposiciones matemáticas sólo para inferir de las proposiciones que no pertenecen a la matemática otras proposiciones, las cuales, igualmente, no pertenecen a las matemáticas.

(En filosofía, la cuestión ‘¿Con qué fin usamos propiamente tal palabra, tal proposición?', lleva siempre a resultados valiosos). ${ }^{2}$

Según el primer Wittgenstein, la aritmética se entiende mejor si concebimos las ecuaciones numéricas como reglas de inferencia. La sugerencia es que tratemos una ecuación como algo que avala la transición de una proposición empírica que contiene una expresión ubicada en el lado izquierdo de la ecuación a otra proposición empírica en la que la expresión se sustituye por lo que está ubicado en el lado derecho de la ecuación. No debe asumirse, sin embargo, que el Tractatus propone una reducción de la aritmética a la lógica. Pues, aunque toma tanto las ecuaciones como las tautologías por seudoproposiciones (o proposiciones que muestran las propiedades formales del simbolismo), las tautologías se consideran como casos límite de proposiciones genuinas, ${ }^{3}$ mientras las ecuaciones contienen dos expresiones numéricas conectadas por el signo de identidad.

${ }^{1}$ Véase Ludwig Wittgenstein, Tractatus Logico-Philosophicus. Londres, Routledge, $1922,6.2,6.21,6.22,6.23$ y 6.24 .

${ }^{2}$ Ibid., 6.211.

${ }^{3}$ Esto es, proposiciones cuya última columna de la tabla de verdad contiene sólo verdades como valor de verdad. 
La objeción estándar a esta manera de concebir a la aritmética es que no cubre todo lo que conocemos como la aritmética de primer orden de PeanoDedekind; excluye, por ejemplo, desigualdades y enunciados con cuantificadores indefinidos. La idea de que la aritmética de las ecuaciones numéricas es todo lo que necesitamos para la aplicación extramatemática de la aritmética es obviamente muy poco satisfactoria. Pero no tiene caso evaluar este punto aquí, ya que Wittgenstein lo abandonó posteriormente.

Lo que me parece relevante es el descubrimiento temprano de que en las matemáticas y en la lógica estamos esencialmente aplicando reglas; Wittgenstein nunca abandonó esta idea en su filosofía posterior. ${ }^{4}$ Es cierto, sin embargo, que su concepción de lo que es una regla cambia radicalmente en la fase tardía. En el Tractatus, por ejemplo, las ecuaciones y las tautologías se encuentran entre las reglas de la sintaxis lógica de nuestro lenguaje, esto es, reglas para manipular signos; ${ }^{5}$ en este sentido, son ellas metalingüísticas.

${ }^{4}$ En el periodo del Tractatus, y en el periodo intermedio, los términos funcionales lógicos y aritméticos eran explicados como reglas de sintaxis. Para usar la terminología del Tractatus, ellos expresaban operaciones, $i$. e. algoritmos definidos para manipular signos. Similarmente, las oraciones lógicas y aritméticas representaban reglas de inferencia. Se consideraba que las ecuaciones numéricas, por ejemplo, correspondían a reglas de sustitución. En la fase tardía, en contraste, las reglas lingüísticas son, en primer lugar, regularidades empíricas que un intérprete discierne en el comportamiento de un hablante. En segundo lugar, son generalizaciones universales (comparables con las leyes naturales) que presenta un intérprete para abarcar esas regularidades empíricas. Finalmente, las reglas son regularidades normativas que el intérprete propone para explicar el comportamiento de su interpretado, guiado aquél por las condiciones de corrección que supuestamente conoce. Los siguientes pasajes, por ejemplo, confirman que Wittgenstein consideraba las actividades de inferir y calcular como actividades de seguimiento de reglas en los tres sentidos mencionados: "Las leyes de la lógica son efectivamente la expresión de 'hábitos de pensamiento', pero también lo son del hábito de pensar. Esto es, puede decirse que muestran cómo piensan los seres humanos, y también lo que los seres humanos llaman 'pensar'" (L. Wittgenstein, Remarks on the Foundations of Mathematics. 3a. ed. Nueva York, Harper Torchbooks, 1971, p. 89).

“¿Y qué si dijéramos que las proposiciones matemáticas son profecías, en este sentido: que producen el resultado que obtendrán los miembros de una sociedad que han aprendido esta técnica, en concordancia con otros miembros de la sociedad? '25 $\times$ 25 = 625' significaría que los hombres, si juzgamos que ellos obedecen las reglas de multiplicación, obtendrán el resultado 625 cuando multiplican $25 \times 25$. -Está más allá de la duda que ésta es una predicción correcta; y también lo está que los cálculos se basan en tales predicciones" (ibid., pp. 192-193).

"El carácter dual de la proposición matemática -como ley y como regla" (ibid., p. 235).

Para abundar en esto, véase Silvio Pinto, Wittgenstein, Meaning and Mathematics. Tesis. Londres, Universidad de Londres, 1998.

${ }^{5}$ Véase L. Wittgenstein, Tractatus Logico-Philosophicus, 3.331, 241, 6.02, 6.124 y 6.126. Wittgenstein las llama Zeichenregeln. 
Según Carnap, hay dos tipos de reglas sintácticas: reglas de formación y reglas de transformación. ${ }^{6}$ Las primeras gobiernan la formación de expresiones complejas a partir de expresiones subsentenciales, más simples, así como de expresiones más complejas a partir de las oraciones menos complejas que las componen. La obtención de términos singulares más complejos a partir de términos más simples, por ejemplo, se trata con la formación de reglas del primer tipo. El Tractatus ofrece un ejemplo de la formación de reglas del segundo tipo: las operaciones de verdad $-i$. e., la aplicación de las operaciones lógicas de funciones de verdad-sobre una o más proposiciones. Wittgenstein representa las operaciones de verdad de modo bastante general como la aplicación de uno de los operadores de Scheffer - el operador no-y: $N(\xi, \zeta)$ - a una clase de proposiciones; produce la conjunción de la negación de cada proposición en la clase. ${ }^{7}$ La definición tractariana de la función del sucesor aritmético en términos de la reiteración de una operación es otro ejemplo de reglas de formación. Se formula como sigue

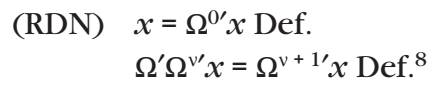

Las reglas de transformación, por otro lado, gobiernan las inferencias; legislan sobre qué proposiciones se siguen de qué otras proposiciones. El punto de vista del Tractatus es que las proposiciones de la lógica (tautologías) son de hecho reglas de inferencia encubiertas. El siguiente pasaje, por ejemplo, es explícito al respecto:

La proposición con significado dice algo, y su prueba muestra que esto es así; en lógica toda proposición es la forma de una prueba.

Toda proposición de la lógica es un modus ponens presentado en signos. (Y el modus ponens no puede ser expresado por una proposición). ${ }^{9}$

La idea es que cada tautología es una regla de inferencia expresada de tal modo que la hace parecer una proposición genuina. Así, el modus ponens

\footnotetext{
${ }^{6}$ Rudolf Carnap, The Logical Syntax of Language. Londres, Keagan Paul, Trench, Trubner, 1937.

${ }^{7}$ L. Wittgenstein, Tractatus Logico-Philosophicus, 5.5-5.51.

8 ' $\Omega$ ' designa una operación arbitraria y ' $x$ ' designa la expresión a la que se aplica; ' $x$ ' puede ser una oración, pero también un nombre propio o alguna otra expresión. (Véase ibid., 6.02).

${ }^{9}$ Ibid., 6.1264.
} 
puede expresarse como una tautología, la así llamada condicionalización del modus ponens

$$
(\mathrm{CMP}) \quad((p \rightarrow q) \wedge p) \rightarrow q
$$

La apreciación de Wittgenstein de que la lógica consiste esencialmente en una colección de reglas de inferencia es ahora familiar en las exposiciones recientes de la lógica en términos de deducción natural. El primero en incorporar esta apreciación en un sistema de deducción natural fue Gerhard Gentzen ${ }^{10}$ trece años después del Tractatus.

La equivalencia tractariana entre tautologías y reglas de inferencia provee la clave para explicar la conexión entre la lógica y la realidad. Según el primer Wittgenstein, el lenguaje sólo puede representar la realidad debido a que comparte su forma lógica con el mundo. Esto significa que la sustancia del mundo (los objetos tractarianos) ha de poseer las mismas posibilidades combinatorias para formar estados de cosas que las que sus respectivos nombres deben poseer para formar proposiciones elementales. ${ }^{11}$ También significa que las relaciones lógicas entre proposiciones más complejas y simples, que según el Tractatus muestran las tautologías y las contradicciones, deben también mantenerse entre sus respectivos hacedores de verdad, los hechos que las hacen verdaderas. ${ }^{12}$ Así, supongamos que $p$ y $q$ son dos proposiciones. Dos relaciones lógicas entre ellas y su conjunción pueden expresarse mediante las siguientes tautologías o por sus correspondientes reglas de inferencia:

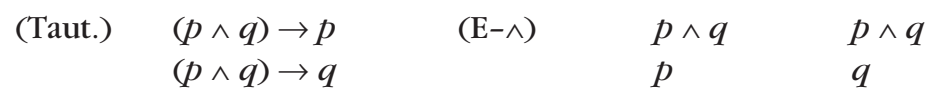

Ahora bien, llámense hacedores de verdad de $p \wedge q$, de $p$ y de $q ; P \wedge Q, P$ y $Q$, respectivamente. $P \wedge Q-i$. $e$. la obtención simultánea del hecho de que $p$ y del hecho de que $q-$ y $P$ se relacionan de tal modo que si se da el primero, el segundo debe darse también. Similarmente, para $P \wedge Q$ y $Q$.

Pero la lógica comprende, de acuerdo con el autor del Tractatus, no sólo reglas de inferencia. Como ya hemos sugerido más arriba, para él los términos lógicos también expresan reglas metalingüísticas. A diferencia de Frege, quien las asimilaba a expresiones conceptuales, Wittgenstein sostenía que ellas correspondían a operadores de verdad; las consideraba reglas para la cons-

\footnotetext{
${ }^{10}$ Véase G. Gentzen, "Untersuchungen über logische Schliessen", en M. Szabo, The Collected Papers of Gerhard Gentzen. Amsterdam, North Holland, 1969.

${ }^{11}$ Véase L. Wittgenstein, Tractatus Logico-Philosophicus, 3.21.

12 Ibid., 5.122-5.124.
} 
trucción de signos proposicionales complejos a partir de signos más simples. Los términos lógicos en la estructura del Tractatus expresan lo que Carnap llamaría reglas de formación. La formulación tractariana de la forma general de la operación de verdad es esta:

$$
\text { (FGOV) }[\underline{p} ; \underline{\xi} ; N(\underline{\xi})] \cdot{ }^{13}
$$

Así, la implicación material, usualmente representada por la oración " $\mathrm{p} \rightarrow \mathrm{q}$ " sería equivalente a la siguiente operación de verdad:

(IM) $[(p, q) ;(\xi, \zeta) ; N(N(N(\xi, \xi), \zeta), N(N(\xi, \xi), \zeta))]^{14}$

Las proposiciones de la aritmética se identifican con las reglas de inferencia. Esto se corrobora por la primera cita de este trabajo, así como por el siguiente pasaje: "Pues las ecuaciones expresan la sustituibilidad de dos expresiones, y nosotros procedemos de un número dado de ecuaciones a otras nuevas ecuaciones, sustituyendo las expresiones por otras, de acuerdo con las ecuaciones".$^{15}$

Según el Tractatus, las ecuaciones son reglas para la sustitución de expresiones. En esa obra, juegan el mismo papel que las definiciones, las cuales también son concebidas como reglas de sustitución. Veamos lo que dice el autor acerca de las definiciones:

Si yo uso dos signos con uno y el mismo significado, lo expreso poniendo entre los dos el signo "=".

" $a=b$ " significa, pues, que el signo "a" es reemplazable por el signo "b".

(Si yo introduzco en una ecuación un nuevo signo "b" para determinar que debe sustituir un signo "a" ya conocido, escribo (como Russell) la ecuación -definición - en la forma: "a = b def." La definición es una regla del signo). ${ }^{16}$

${ }^{13}$ El subrayado indica el conjunto de todas las proposiciones a las que se aplica el operador $N$. El primer término de la expresión entre corchetes representa el conjunto de las proposiciones iniciales. El segundo término indica el conjunto de argumentos del operador $N$. Finalmente, el tercer término denota el tipo de transformación que efectúa $N$ sobre los argumentos.

${ }^{14} N(\xi, \zeta)$ designa el operador no-y generalizado que se aplica a dos signos proposicionales.

${ }^{15}$ L. Wittgenstein, Tractatus Logico-Philosophicus, 6.24 .

${ }^{16}$ Ibid., 4.241. 
Las expresiones acerca de cuya sustitución legislan las ecuaciones numéricas son los términos numéricos. Así, por ejemplo, la proposición genuina expresada por "las seis manzanas que están sobre la mesa son Cox"17 implica las proposiciones que corresponden a las oraciones "tres veces dos manzanas sobre la mesa son Cox" y "las cuatro más dos manzanas sobre la mesa son Cox", ${ }^{18}$ de acuerdo con las reglas de sustitución expresadas por " $3 \times 2=6$ " y “4 + 2 = 6", respectivamente. El primer Wittgenstein sostenía que un término numérico expresa el exponente de una operación. ${ }^{19}$ Permítaseme decir algo más acerca de la conexión entre expresiones y operaciones aritméticas en el Tractatus.

La definición recursiva en el Tractatus: $6.02(\mathrm{RDN})$ muestra cómo reducir la función de sucesor a la noción de operación: la aplicación de una operación $(\Omega)$ al argumento resultante de $n$ aplicaciones de $\Omega$ a un argumento $x$ se define como el sucesor de $n$ aplicaciones de $\Omega$ a $x$. RDN también efectúa una reducción del primero, y por tanto de todos los números naturales a contadores de las reiteraciones de operaciones. Así, el cero no cuenta ninguna aplicación de la operación $\Omega$ al argumento $x$. El número uno corresponde a la aplicación de $\Omega$ a cero aplicaciones de $\Omega$ a $x$; cuenta el sucesor de cero aplicaciones de $\Omega$ a $x$; el dos se identifica con la aplicación de $\Omega$ a una aplicación de $\Omega$ a $x$; cuenta el sucesor de una aplicación de $\Omega$ a $x$; el tres se identifica de modo similar, así como el cuatro, el cinco, y el resto de los números naturales. Con ayuda de definiciones adecuadas de la adición (DA) y de la multiplicación (DM) de reiteraciones de operador, RDN permite una demostración del carácter recursivo de todas estas operaciones nuevas. Las dos definiciones requeridas son las siguientes:

(DA) $\Omega^{v^{\prime}} \Omega^{\mu^{\prime}} x=\Omega^{v+\mu^{\prime}} x$ Def.

(DM) $\left(\Omega^{v}\right)^{\mu^{\prime}} x=\Omega^{v \times \mu^{\prime}} x$ Def. $^{20}$

Las definiciones RDN, DA y DM pueden entonces usarse para demostrar el isomorfismo entre la estructura estándar de los números naturales con sucesor, adición y multiplicación, y la estructura de las operaciones tractarianas

${ }^{17}$ Imagínese que esta oración es el resultado de cinco aplicaciones de la operación que genera la siguiente serie de oraciones: "la (una) manzana sobre la mesa es Cox", "las dos manzanas sobre la mesa son Cox", "las tres manzanas sobre la mesa son Cox", y así sucesivamente.

${ }^{18}$ Suponiendo, desde luego, que estas oraciones puedan expresar proposiciones tractarianas genuinas.

${ }^{19}$ Recuérdese la definición tractariana de la serie de los números naturales (RDN).

${ }^{20}$ L. Wittgenstein, Tractatus Logico-Philosophicus, 6.241. 
con sus respectivas operaciones de sucesor, adición y multiplicación sobre los operadores (dadas por RDN, DA y DM, respectivamente). Así, podría demostrarse que todas las ecuaciones numéricas de la forma " $m+n=p$ " y " $m \times n=q$ " son equivalentes a las seudoproposiciones expresadas por “ $\Omega^{m+n^{\prime}} x=\Omega^{p^{\prime}} x$ " y “ $\Omega^{m \times n^{\prime}} x=\Omega^{q} x^{\prime}$. ${ }^{21}$

Y en general cada ecuación numérica tendría su correspondiente seudoproposición en la estructura tractariana de operaciones.

Mencioné un par de párrafos atrás la pretensión tractariana de que las ecuaciones numéricas son reglas de sintaxis del lenguaje. Ahora podemos ver más claramente la razón de esto. Las operaciones de sucesión, adición y multiplicación sobre las operaciones que Wittgenstein asocia con sus respectivas contrapartes aritméticas son ellas mismas reglas metalingüísticas; son reglas para generar expresiones nuevas (términos complejos o signos proposicionales) a partir de expresiones viejas. Así, la aplicación del operador- $N$ al resultado de su aplicación a una proposición $p$ nos permite construir el signo proposicional " $N^{1+1}(p, p)$ " (о " $N^{2}(p, p)$ ") a partir de “ $N(\xi, \zeta)$ " y " $p$ ". Similarmente, las identidades entre los resultados de las diferentes reiteraciones de una operación son ellas mismas reglas para la sustitución de una expresión por otra. Así, el signo seudoproposicional, que según el Tractatus abrevia " $2+3=5$ ", muestra el carácter de ser intersustituible de las expresiones " $\Omega^{2+3} p$ " y " $\Omega^{5 \prime} p$ ".

\section{La generalidad de una regla}

Otro rasgo relevante de las reglas tractarianas es su generalidad. En el libro, Wittgenstein afirma que las reglas lógicas y matemáticas poseen generalidad esencial, en oposición a la generalidad accidental que se atribuye a las proposiciones empíricas. Él caracteriza la generalidad accidental como: a) la especificación de un conjunto de proposiciones obtenidas al sustituir cada posible argumento de una función proposicional en el lugar de su variable, y b) la aplicación simultánea de los operadores de verdad apropiados a todas las proposiciones en el conjunto. ${ }^{22}$ Un ejemplo de una oración que expresa una proposición accidentalmente general es: "Todos los hombres son mortales". ${ }^{23}$ La función proposicional que corresponde a esta oración es la siguiente: $\boldsymbol{s i} \mathbf{x}$ es un hombre, entonces $\mathrm{x}$ es mortal. Suponiendo que todos los posibles argu-

${ }^{21}$ Este punto fue claro para mí después de leer la primera parte del libro de Frascolla sobre Wittgenstein.

${ }^{22}$ Véase L. Wittgenstein, Tractatus Logico-Philosophicus, 5.52-5.521.

${ }^{23}$ Ibid., 6.1232. 
mentos de esta función proposicional sean personas, podríamos formar, según la instrucción (a), el siguiente conjunto de proposiciones: si Sócrates es un hombre, entonces Sócrates es mortal; si Platón es un hombre, entonces Platón es mortal; si Aristóteles es un hombre, entonces Aristóteles es mortal, y así sucesivamente. Según la instrucción (b), para formar una oración universalmente generalizada debemos aplicar el operador de conjunción generalizada simultáneamente a todas las proposiciones de este conjunto. ${ }^{24}$

Por otro lado, la generalidad esencial se concibe como la marca de una operación. Así, por ejemplo, la forma general de una proposición se presenta en el Tractatus como una operación -el operador generalizado no- $y$ - por medio de la cual se pueden generar todas las funciones de verdad de las proposiciones elementales. En el aforismo 6 esta operación se expresa como sigue:

$$
\text { (GFP) }[p, \xi, N(\xi)] \cdot{ }^{25}
$$

Similarmente, la forma general de un número natural es introducida como una operación (el operador de la sucesión) para producir todos los números naturales. Aparece en los aforismos 6.03 y 6.02, respectivamente:

(GFN) $[0, \xi, \xi+1]$ que abrevia $\left[\Omega^{0 \prime} x, \Omega^{{ }^{\prime}} x, \Omega^{v+1} x\right] \cdot{ }^{26}$

El primer Wittgenstein mantuvo que la generalidad de las operaciones aritméticas (sucesión, adición, multiplicación, etcétera) y lógicas (negación, conjunción, disyunción, etcétera) reside en su carácter recursivo. Esto es, cualquier término numérico es el resultado de un número finito de aplicaciones de las operaciones matemáticas. Similarmente, cualquier signo proposicional es el resultado de un número finito de aplicaciones de las operaciones lógicas. La recursividad de las operaciones lógicas (aritméticas) es lo que permite que puedan decidirse las cuestiones acerca de si una expresión es un signo proposicional o un término numérico. Este rasgo de las operaciones aritméticas es también lo que garantiza la existencia de una prueba canónica para cualquier ecuación numérica.

${ }^{24}$ Para obtener la conjunción de todas las proposiciones anteriores basta aplicar la versión generalizada del operador de Scheffer a cada una de sus respectivas negaciones. Como vimos antes, este es el operador de verdad que Wittgenstein usa en el Tractatus (L. Wittgenstein, Tractatus Logico-Philosophicus, 5.502-5.51, 5.52 y 66.01). Puede probarse fácilmente que este único operador es suficiente para expresar toda función de verdad de las proposiciones elementales.

25 Ésta es también la forma general de una operación de verdad (FGOV).

${ }^{26}$ Véase más arriba para una explicación de la equivalencia entre ambos. 
De modo que, según el Tractatus, la generalidad matemática y lógica es una propiedad de las operaciones, $i$. e. su recursividad. No tiene nada que ver con cuantificación. Recuérdese que el primer Wittgenstein quería que las oraciones de la lógica y las matemáticas fueran todas decidibles; en el sistema del Tractatus no había lugar para oraciones aritméticas indefinidamente cuantificadas.

Esta explicación de la distinción tractariana entre generalidad accidental y generalidad esencial -cuantificacional y recursiva (por falta de un mejor adjetivo), respectivamente- puede también, pienso, arrojar luz sobre el siguiente aforismo enigmático: "La teoría de las clases es superflua en matemáticas. Esto está en conexión con el hecho de que la generalidad de la cual tenemos necesidad en matemáticas no es accidental". ${ }^{27}$

En el primer capítulo de su libro sobre Wittgenstein, Pasqualle Frascolla ${ }^{28}$ mantiene que la objeción tractariana a la reducción russelliana de la aritmética a la teoría de las clases es que ésta analiza proposiciones que poseen generalidad esencial en términos de proposiciones cuya generalidad es meramente accidental. Según Frascolla, Russell habría traducido proposiciones de la aritmética de primer orden, que son verdaderas en todos los mundos posibles, a proposiciones de la teoría de conjuntos, que son sólo contingentemente verdaderas. Me parece que éste es un gran error de interpretación de la objeción wittgensteiniana al proyecto de Russell.

Es verdad que para Wittgenstein el proyecto estaba mal encaminado porque la lógica que asumía como la base para reducir la aritmética a la lógica incluía proposiciones como los axiomas de elección, infinitud y reductibilidad, cuya generalidad no es recursiva o esencial. Pero esto por sí mismo no es una objeción, para Wittgenstein, porque Russell pretendía que las proposiciones de la lógica del Principia Mathematica ${ }^{29}$ fueran verdaderas de ciertos aspectos de este mundo. Pues, aun si ellas fueran verdaderas, eso sería una mera contingencia, puesto que su generalidad no es esencial. La objeción de Wittgenstein no es entonces una objeción a la teoría de conjuntos como tal, sino sólo a la teoría russelliana de las clases como éste la concebía, esto es, como una teoría verdadera de los aspectos más generales del mundo.

Más aún, puesto que los axiomas de la teoría de conjuntos no poseían el tipo de generalidad que Wittgenstein consideraba como la marca de las proposiciones aritméticas y lógicas -su recursividad-, él pensaba que la teoría

${ }^{27}$ L. Wittgenstein, Tractatus Logico-Philosophicus, 6.031 .

${ }_{28}$ Pasqualle Frascolla, Wittgenstein's Philosophy of Mathematics. Londres, Routledge, 1994.

${ }^{29}$ Bertrand Russell, Principia Mathematica. 
de conjuntos no se requería para hacer espacio a la aritmética que es indispensable para la mayoría de las aplicaciones prácticas (la aritmética de las ecuaciones numéricas). Según el autor del Tractatus, para ello bastaba la noción de reiteración de una operación; y creía que la aritmética y la lógica consistían en proposiciones decidibles. La concepción tractariana de la aritmética contiene las semillas de la aritmética recursiva primitiva de Skolem. ${ }^{30}$

\section{¿Podemos eliminar los objetos matemáticos?}

Los críticos se han quejado de que la concepción tractariana de las matemáticas deja fuera su parte más importante, i. e. la parte indecidible. Más aún, otra objeción popular es que incluso las aplicaciones básicas de la aritmética en la física requieren oraciones aritméticas con cuantificadores indefinidos. ${ }^{31}$ De nuevo, no enfrentaré éstas objeciones porque la perspectiva tractariana no corresponde a la concepción del Wittgenstein maduro.

Sin embargo, vale la pena considerar en qué medida su primera concepción de las matemáticas elimina a los objetos matemáticos. En la discusión siguiente hay dos aspectos de la concepción tractariana que se vuelven relevantes. El primero radica en la idea de que los que parecen ser términos ma-

${ }^{30}$ Véase T. Skolem, "The Foundations of Elementary Arithmetic established by means of the Recursive Mode of Thought, without the Use of Apparent Variables ranging over Infinite Domains", en J. van Heijenoort, From Frege to Gödel. A Source Book in Mathematical Logic, 1879-1931. Cambridge, Universidad de Harvard, 1967. Su perspectiva se proponía mostrar que es posible desarrollar la aritmética sin usar nociones de teoría de conjuntos. Se volvió conocida como aritmética recursiva primitiva debido a su utilización de la noción de recursión -tanto para generar expresiones aritméticas como para verificar la corrección de las fórmulas aritméticas- como la noción básica de la aritmética. La aritmética de Skolem es mucho más débil que la aritmética de primer orden de Peano, y de hecho, también es más débil que la aritmética intuicionista. Esto se debe a que limita el poder expresivo del discurso aritmético a lo que puede decirse sin el uso de cuantificadores indefinidos. De ahí que no se requieran nociones de teoría de conjuntos. Uno de los méritos de la aritmética recursiva primitiva es que ilustra una manera de evitar las paradojas que afligieron las perspectivas de la teoría de conjuntos sin recurrir a la dificultosa teoría de los tipos de Russell. A pesar de su poder expresivo más débil, la aritmética de Skolem fue aplaudida por Hilbert (D. Hilbert y P. Bernays, Die Grundlagen der Mathematik, vol. 1. Berlín, Springer Verlag, 1934), quien la consideraba como una herramienta perfecta con la cual probar la consistencia de la aritmética de primer orden plenamente desarrollada; para Hilbert, representaba la mejor ilustración de un modo finitista de pensamiento.

${ }^{31}$ Esta segunda objeción me fue presentada oralmente por Crispin Wright, pero también puede encontrarse en la crítica de Hilary Putnam al nominalismo matemático. Véase H. Putnam, Philosophy of Logic. Nueva York, Harper Torchbooks, cap. v. 
temáticos singulares están definidos mediante una operación recursiva con signos; especifican el número de reiteraciones de una operación sobre los signos. El segundo aspecto relevante es la tesis de que la generalidad matemática no reside en los cuantificadores sino en la recursividad de las reglas matemáticas. Pensemos ahora en oraciones de la aritmética aplicada en las que las expresiones que contienen palabras-números parecen ser términos singulares, o requerir cuantificación de los números. Tómese, por ejemplo, la oración "el número de preguntas a contestar es tres" $\left(A_{3}\right)$. Una manera de lidiar con oraciones como ésta es tratar la ocurrencia de palabras-números como adjetivaciones. Esto es, parafraseando la oración anterior, "hay exactamente tres preguntas a contestar". Y sabemos que las ocurrencias de adjetivaciones en las palabras-números puede ser parafraseada en términos de cuantificadores, funciones de verdad e identidad. ${ }^{32}$

La estrategia tractariana para analizar estas oraciones parece coincidir con la anterior. En dos de los pocos pasajes en los que se menciona explícitamente la aplicación de palabras-números, encontramos que Wittgenstein afirma:

Por ejemplo, en la proposición "Hay dos objetos que..." [el que la palabra "objeto" se use correctamente está expresado] por " $(\exists x, y) \ldots$..."

Si queremos expresar en el simbolismo lógico la proposición general "b es el sucesor de a", necesitaremos una expresión para el término general de la serie formal:

$a R b$,

$(\exists x): a R x, x R b$,

$(\exists x, y): a R x . x R y, y R b$,

$\cdots$

El término general de una serie formal sólo puede expresarse por una variable, pues el concepto "término de esta serie formal" es un concepto formal. ${ }^{33}$

${ }^{32}$ Esto es, en lugar de atribuir a las palabras-número el papel de términos singulares, podemos interpretarlas como cuantificadores numéricos. Así, una oración como "Júpiter tiene cuatro lunas" puede parafrasearse como "hay exactamente cuatro cosas que satisfacen el concepto luna de Júpiter" y no como "el número que pertenece al concepto luna de Júpiter es el cuatro", que era la paráfrasis preferida por Frege. En lenguaje estándar de primer orden, los cuantificadores numéricos pueden definirse recursivamente como sigue:

$$
\begin{aligned}
& \left(\exists_{0} x\right)(F x)=_{\mathrm{df}} \neg(\exists x)(F x) \\
& \left(\exists_{\mathrm{n}+1} x\right)(F x)=_{\mathrm{df}}(\exists x)\left(F x \wedge\left(\exists_{\mathrm{n}} y\right)(F y \wedge \neg(y=x))\right. \\
& { }^{33} \text { L. Wittgenstein, Tractatus Logico-Philosophicus, } 4.1272 \text { y } 4.1273 .
\end{aligned}
$$


Las citas sugieren que el autor del Tractatus habría tratado el numeral en $A_{3}$ como un cuantificador numérico. Más evidencia de la inclinación reduccionista de Wittgenstein proviene del siguiente pasaje, que data del comienzo del periodo intermedio:

La proposición "Hay dos cosas con la propiedad $f$ " puede representarse como sigue:

$(\exists x, y) \cdot f x \cdot f y \cdot \sim(\exists x, y, z) \cdot f x \cdot f y \cdot f z$.

Aquí el número 2 ocurre como un rasgo de representación de nuestro simbolismo. ${ }^{34}$

La segunda cita del Tractatus también aclara el sentido en el que se dice de un numeral que simboliza el exponente de una operación. Tómese, por ejemplo, la siguiente serie de oraciones:

$$
\begin{array}{ll}
\left(A_{0}\right) & \sim(\exists x)(f x) \\
\left(A_{1}\right) & (\exists x)(f x) \cdot \sim(\exists x, y)(f x, f y) \\
\left(A_{2}\right) & (\exists x, y)(f x \cdot f y) \cdot \sim(\exists x, y, z)(f x, f y \cdot f z) \\
\left(A_{3}\right) & (\exists x, y, z)(f x \cdot f y \cdot f z) . \sim(\exists x, y, z, w)(f x, f y \cdot f z \cdot f w) \\
\ldots &
\end{array}
$$

en donde ' $f x$ ' abrevia el predicado ' $x$ es una pregunta a contestar'. Según el primer Wittgenstein, lo que ata todas estas operaciones entre sí es que la forma de cada una de ellas puede obtenerse a partir de la forma de la oración previa mediante la aplicación de la operación $(\Omega)$, que añade el mismo predicado con una variable diferente a la primera y segunda oraciones de la conjunción. El numeral que aparece en cada oración identifica el número de aplicaciones de $\Omega$ a $A_{0}$. Esto está en conformidad, e ilustra, la caracterización tractariana de la forma general de un número natural como (GFN: $\left[\Omega^{0 \prime} x, \Omega^{{ }^{\prime}} x\right.$, $\left.\left.\Omega^{v+1^{\prime}} x\right]\right){ }^{35}$

Ahora pensemos un ejemplo de una oración de matemáticas aplicadas que parece cuantificar sobre los números. Tomemos el caso, propuesto por Hilary Putnam, ${ }^{36}$ de la oración (Q) "la distancia $d_{\mathrm{ab}}$ es $r_{1} \pm r_{2}$ ", donde $r_{1}$ y $r_{2}$ son números racionales arbitrarios. Según Putnam, equivale a

${ }^{34}$ F. Waismann, Wittgenstein und der Wiener Kreis. Fráncfort/Oxford, Suhrkamp/ Basil Blackwell, 1967, p. 223.

35 También arroja luz sobre la nota 12.

${ }^{36} \mathrm{El}$ contexto es el de mostrar que nuestras mejores teorías físicas no pueden ser plenamente nominalistas. Putnam arguye que una oración como, por ejemplo, Q, que debe ser parte de nuestra mejor teoría de la mecánica, cuantifica sobre objetos matemáticos. Véase H. Putnam, Philosophy of Logic, cap. v. 
(R) $\quad(\forall a)(\forall b)\left(\exists d_{a, b}\right)\left(\exists r_{1}\right)\left(\exists r_{2}\right)\left(d_{a, b}=r_{1} \pm r_{2}\right)$, donde $a$ y $b$ son puntos espaciales.

Putnam mantiene que $\mathrm{Q}$, como $\mathrm{R}$, cuantifica sobre números racionales y funciones de puntos espaciales para los números racionales. Voy a posponer la discusión de Q para un análisis más amplio de la explicación de Wittgenstein, puesto que el Tractatus no menciona los números racionales o reales, ni eso que Hartry Field llama entidades matemáticas impuras, tales como las distancias. ${ }^{37}$ Consideremos, en su lugar, un análogo de Q para los números naturales, $i$. e e la oración (Q') "la cardinalidad de la extensión $u$ para el predicado ' $f(x)$ ' $\left(C_{u}\right)$ es $n$ ”. Si Putnam tiene razón, Q' debería ser equivalente a

$\left(\mathrm{R}^{\prime}\right)(\forall u)\left(\exists C_{u}\right)(\exists n)\left(C_{u}=n\right)$, donde $n$ es un número natural, y $C$ una función de extensiones de los predicados a los números.

Pero ya hemos visto que $Q^{\prime}$ también es equivalente a "hay $n$ objetos que satisfacen el predicado ' $f(x)$ '”, que, ni contiene números-palabras como términos singulares, ni cuantifica sobre números. Más aún, no contiene un término que designe una función de cardinalidad (otra entidad matemática impura). La generalidad de $\mathrm{Q}^{\prime}$, creo que diría Wittgenstein, consiste en representar (standing for) la siguiente serie de formas oracionales:

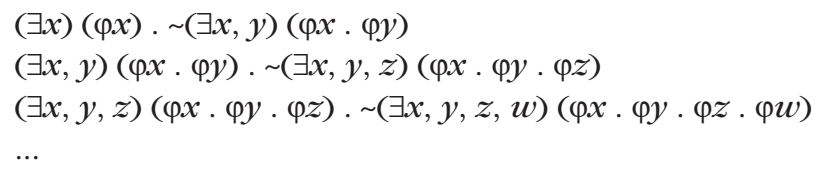

donde $\varphi x$ es una variable para predicados de una posición. La generalidad de Q' corresponde a la recursividad de la operación que genera la serie anterior.

Hay por supuesto ejemplos más complicados de la aparente cuantificación sobre los números y sobre otras entidades matemáticas impuras. Pospondré su discusión para otra ocasión. Para mi propósito es suficiente por el momento concluir que el Tractatus logra eliminar la referencia y la cuantificación sobre objetos matemáticos para los casos más simples.

${ }^{37}$ Una distancia, según Field, es una función, de pares de puntos espaciales a números racionales, que satisface la métrica euclideana. A esto se debe que se le llame una entidad matemática impura. Véase H. Field, Science without Numbers. Princeton, Universidad de Princeton, pp. 9-10. 


\section{La epistemología de la aritmética y la lógica}

El último tema a tratar concierne a la posición del Tractatus en torno a la cuestión de nuestro conocimiento de la aritmética y la lógica. Según el primer Wittgenstein, nuestro acceso a las ecuaciones y a las tautologías es a priori. En cuanto a la aritmética, son elocuentes los siguientes pasajes:

Es posible calcular si una proposición pertenece a la lógica calculando las propiedades lógicas del símbolo.

Y esto hacemos cuando "probamos" una proposición lógica. Pues sin preocuparnos del sentido y significado formamos la proposición lógica desde otras, según meras reglas simbólicas.

La prueba de una proposición lógica consiste en esto, en obtenerla de otras proposiciones lógicas por la aplicación sucesiva de ciertas operaciones con las cuales se continúa obteniendo de las primeras proposiciones nuevas tautologías. ${ }^{38}$

La prueba en lógica es sólo un expediente mecánico para facilitar el reconocimiento de la tautología, cuando es complicada. ${ }^{39}$

El Tractatus también aboga por otro método mecánico para reconocer tautologías, a saber: el de construir una tabla de verdad de la expresión en cuestión. ${ }^{40}$ Por lo menos en los casos en los que la tabla de verdad tiene un número finito de líneas, el método permite llegar a una decisión. De nuevo, el método que utilicemos para mostrar que una cierta expresión es una tautología sólo requiere una comprensión de las reglas sintéticas del lenguaje. Éstas pueden ser operaciones lógicas que transforman una tautología en otra (reglas de inferencia); pero también pueden ser algoritmos para construir tablas de verdad. En cualquier caso, nuestra compresión de las operaciones lógicas debe ser a priori. Como Wittgenstein dice en un famoso pasaje:

La "experiencia”, de la que tenemos necesidad para entender la lógica, no es que algo ocurra de tal y tal modo, sino que algo es; pero esto no es experiencia.

La lógica precede a toda experiencia -que algo es así. Es antes que el cómo, no que el qué. ${ }^{41}$

${ }^{38}$ L. Wittgenstein, Tractatus Logico-Philosophicus, 6.126.

39 Ibid., 6.1262.

${ }^{40}$ Cf. ibid., 4.431-4.4661 у 6.1203 .

${ }^{41}$ Ibid., 5.552. 
La lógica no podría conocerse a posteriori, piensa el autor del Tractatus, porque el conocimiento de la lógica está ya presupuesto cuando comparamos una proposición con la realidad. El conocimiento lógico se ocupa, por ejemplo, de cuáles son los estados de cosas posibles, cuáles proposiciones son instanciaciones de cuáles otras, cuáles se siguen de otras, etcétera. Según Wittgenstein, si nuestro acceso a las operaciones y reglas fuera a posteriori, tendría que presuponer una familiaridad previa con las leyes lógicas, por lo que no podría ser a posteriori. ${ }^{42}$

Nuestro conocimiento de las operaciones y ecuaciones matemáticas es también a priori, según el Tractatus. El siguiente pasaje es explícito: "Y que las proposiciones de las matemáticas puedan probarse no significa otra cosa que su corrección es reconocible sin necesidad de comparar, con los hechos, en cuanto a su corrección, lo que ellas expresan". ${ }^{43}$

La razón por la que tales proposiciones deben ser a priori es la misma: el conocimiento de las reglas de sintaxis del lenguaje es un presupuesto para toda aquel que esté en posición de establecer el valor de verdad de cualquier proposición empírica.

Otro punto interesante acerca de la explicación tractariana de la epistemología de la lógica y de la aritmética se relaciona con el papel que le atribuye a la noción de prueba en estas disciplinas. Como lo indican el último y antepenúltimo pasajes, el primer Wittgenstein consideraba la prueba en lógica como un algoritmo meramente mecánico y decidible para reconocer ecuaciones y tautologías complicadas. Tenemos que recurrir a ella porque sin la prueba nuestras mentes sólo podrían percibir las tautologías más simples. El algoritmo, no obstante, nos hace posible captar cualquiera de las infinitamente diversas tautologías y ecuaciones.

${ }^{42} \mathrm{Su}$ argumento es básicamente el mismo que usa Frege. Véase Gottlob Frege, "Logic", en G. Frege, Gottlob Frege: Posthumous Writings. Londres, Blackwell, 1979, p. 3.

${ }^{43}$ L. Wittgenstein, Tractatus Logico-Philosophicus, 6.2321. 